\title{
Teebi-Shaltout syndrome
}

INSERM

\section{Source}

INSERM. (1999). Orphanet: an online rare disease and orphan drug data base. Teebi-

Shaltout syndrome. ORPHA:3291

Teebi-Shaltout syndrome is a rare, genetic, development defect during embryogenesis malformation syndrome characterized by association of characteristic facial features (including abnormal head shape with narrow forehead, hypertelorism, telecanthus, small earlobes, broad nasal bridge and tip, underdeveloped ala nasi, small/wide mouth and high/cleft palate), ectodermal dysplasia (including oligodontia with delayed dentition, slow growing hair and reduced sweating) and skeletal abnormalities including camptodactyly and caudal appendage. Short stature and abnormal palmar creases are additional clinical features. 